\title{
Acoustic neuroma surgery as an interdisciplinary approach: a neurosurgical series of 508 patients
}

\author{
Joerg-Christian Tonn, Hans-Peter Schlake, Roland Goldbrunner, Christian Milewski, \\ Jan Helms, Klaus Roosen
}

\section{Department of Neurosurgery University of Wuerzburg, Josef- Schneider-Strasse 11, D-97080 Wuerzburg, Germany \\ J-C Tonn \\ H-P Schlake R Goldbrunner K Roosen}

Department of Ear Nose and Throat Surgery

J Helms

C Milewski

Correspondence to: $\mathrm{J}-\mathrm{C}$ Tonn

tonn@mail.uni-wuerzburg.de

Received 7 May 1999 and in revised form

16 November 1999 Accepted 7 December 1999

Table 1 Modified ${ }^{\star}$ classification for hearing preservation according to Gardner and Robertson ${ }^{1}$

\begin{tabular}{ll}
\hline Class & PTA $(d B)$ \\
\hline 1 & $0-30$ \\
2 & $31-50$ \\
3 & $51-90$ \\
4 & $91-$-maximum loss \\
5 & no response \\
\hline$\star$ Speech discrimination, an & of the original \\
element of & Gardner/Robertson scale, was \\
omitted as this information \\
was not available in most \\
cases. \\
PTA=Pure tone audiometry.
\end{tabular}

\begin{abstract}
Objectives-To evaluate an interdisciplinary concept (neurosurgery/ear, nose, and throat (ENT)) of treating acoustic neuromas with extrameatal extension via the retromastoidal approach. To analyse whether monitoring both facial nerve EMG and BAEP improved the functional outcome in acoustic neuroma surgery.

Methods-In a series of 508 patients consecutively operated on over a period of 7 years, functional outcome of the facial nerve was evaluated according to the House/Brackmann scale and hearing preservation was classified using the Gardner/Robertson system.

Results-Facial monitoring (396 of 508 operations) and continuous BAEP recording (229 of 399 cases with preserved hearing preoperatively) were performed routinely. With intraoperative monitoring, the rate of excellent/good facial nerve function (House/Brackmann I-II) was $\mathbf{8 8 . 7 \%}$. Good functional hearing (Gardner/ Robertson 1-3) was preserved in $39.8 \%$. Conclusion-Acoustic neuroma surgery via a retrosigmoidal approach is a safe and effective treatment for tumours with extrameatal extension. Functional results can be substantially improved by intraoperative monitoring. The interdisciplinary concept of surgery performed by ENT and neurosurgeons was particularly convincing as each pathoanatomical phase of the operation is performed by a surgeon best acquainted with the regional specialties. (F Neurol Neurosurg Psychiatry 2000;69:161-166)
\end{abstract}

Keywords: acoustic neuroma; intraoperative monitoring; facial nerve; hearing preservation; surgical training

As a result of the rapid improvements and general availability of both neuroradiological imaging (MRI, high resolution CT) and electrophysiological techniques (recording of brainstem auditory evoked potentials (BAEPs)) an increasing proportion of acoustic neuromas are diagnosed at an early stage. Thus, the proportion of patients with minor functional impairment of the facial nerve and hearing is growing steadily. It is this circumstance which challenges the treating physician to meet the expectations of the patient about preservation of nerve function. To meet these expectations an interdisciplinary team was developed at the University Hospital of Wuerzburg Medical School. Patients with isolated intrameatal acoustic neuromas are treated by the ear, nose, and throat (ENT) surgeon, either via a transtemporal or a translabyrinthine approach (depending on the status of cochlear function). In all cases with any extrameatal extension of the neuroma, a team of neurosurgeons and ENT surgeons jointly operated on the patient via the retromastoidal route; to optimise the expertise of both disciplines in the treatment. We present the experience with this concept over a period of almost 7 years from a neurosurgical point of view to discuss its value.

\section{Material and methods}

PATIENTS

From April 1991 until December 1997508 patients (263 men) were operated on for intrameatal and extrameatal acoustic neuromas by the interdisciplinary (neurosurgery/ ENT) method. The age ranged from 14 to 80 years (mean 51.3 years). The tumour size of the extrameatal portion varied from 8 to 40 $\mathrm{mm}$ in diameter. According to a modification of the grading system of Gardner and Robertson, ${ }^{1} 399$ patients $(78.5 \%)$ had good hearing in pure tone audiometry (grade 1-3, table 1). The function of the facial nerve was preoperatively only slightly impaired in some patients with extrameatal tumour extension over $30 \mathrm{~mm}$ in diameter. Facial function was assessed by means of the House/Brackmann scale. $^{2}$

All patients had audiometry and BAEP recording preoperatively. Imaging included routine multiplanar MRI with and without contrast enhancement for tumour delineation and a high resolution (1-2 $\mathrm{mm}$ thickness) skull base CT to evaluate the degree of pneumatisation and excavation of the petrous bone/ internal auditory canal and to relate the extension of the jugular bulb to the internal auditory canal. Plain radiography of the cervical spine was performed to eliminate extensive spondylosis which potentially might lead to nerve root or spinal cord compression due to head rotation and the semisitting position during surgery.

SURGERY

Patients were operated on in a semisitting position, the patient's head being $30^{\circ}$ anteflected and rotated $20^{\circ}$ towards the side of the tumour. Special attention was paid to $90^{\circ}$ hip flexion with the knees raised above the level of the cardiac ventricles (by tilting the operating table backwards) to minimise the risk of venous air embolism. Additionally, a precardial Doppler probe was attached to the chest to detect even minimal venous air bubbles in the right ventricle 
before end expiatory $\mathrm{pCO}_{2}$ decline. In cases where the routine preoperative anaesthesiological examination disclosed cardiopulmonary diseases contraindicating the semisitting position, patients were operated on in the park bench position.

Surgery was performed in all patients using an interdisciplinary approach with resection of the extrameatal portion of the tumour via a retrosigmoidal craniectomy by the neurosurgeon and drilling of the internal auditory canal with removal of the intracanalicular portion by the ENT surgeon. During all phases of tumour dissection, the function of the facial nerve was monitored by EMG of the orbicularis oculi and orbicularis oris muscles (and, depending on the extension of the tumour, nerves V, IX, X, XI, and XII). Since becoming available to our department cochlear function was assessed by online BAEP recordings and, later on, additionally by electrocochleography (ECoG) (see later). Haemostasis was double checked at the end of the procedure by bilateral compression to the jugular veins (in the semisitting position). Any opening of pneumatised cells, either at the internal auditory canal or at the mastoid, was sealed using a muscle patch and fibrin glue. After watertight closure of the dura the craniectomy was sealed with bone cement.

INTRAOPERATIVE MONITORING

Monitoring of cranial nerves V, VII, IX, X, XI, and XII

Monitoring of the cranial motor nerves was performed as described previously in detail. ${ }^{3}$ Briefly, EMG responses from mimic muscles (nerve VII, facial nerve EMG) were recorded using steel needle electrodes inserted subdermally into the orbicular muscles of the eyes and mouth for two channel recordings. For monitoring the trigeminal nerve (nerve $\mathrm{V}$ ), needle electrodes were placed into the masticator muscles ( $\mathrm{m}$ masseter, $\mathrm{m}$ temporalis) and intraorally, under laryngoscopic control needle electrodes enabled EMG recordings of nerves IX, X, and XII.

For electrical stimulation a constant current stimulator (Neurosign ${ }^{\mathrm{TM}} 100$, Magstim Co, UK) was used. In general, square wave pulses of $30 \mathrm{~Hz}$ with a duration of $100 \mu$ s were applied with an intensity of $0.5 \mathrm{~mA}$. If the target nerve was already poorly excitable or residual muscle relaxants could not be excluded, a $3 \mathrm{~Hz}$ stimulation mode was selected.

\section{BAEP Monitoring}

As previously published, ${ }^{3}$ rarefaction click stimuli of supramaximal intensity $(\approx 95 \mathrm{~dB} \mathrm{HL})$ were delivered monaurally through insert earphones (AEARO Co, USA) with disposable foam tips. Broadband contralateral masking ( $30 \mathrm{~dB}$ below stimulus intensity) was presented to the contalateral ear. Stimulation frequency was $20 \mathrm{~Hz}$ with a duration of $150 \mu \mathrm{s} /$ stimulus.

For recordings, a standard protocol was used employing a PC based two channel monitoring device (Intel Pentium ${ }^{\mathrm{TM}}$ ) and the EWACSTM software (based on Microsoft Windows 95 ${ }^{\mathrm{TM}}$ ).
Intraoperative electrocochleography recording To enable simultaneous recordings to be made, identical stimulation variables (stimulation rate of $10-20 \mathrm{~Hz}$ ) were used for BAEP and ECoG with a sufficient quality of signals.

The ECoG was recorded using one of two channels of the above mentioned BAEP device and an Fz to promontory (non-inverting to inverting) electrode array. The ECoG represents a near field potential and the active (promontory) electrode was placed as near as possible to the round window. A Teflon insulated steel needle of $55 \mathrm{~mm}$ length (diameter $0.40 \mathrm{~mm}$ ) served as the promontory electrode. It was inserted by an otologist via the auricular tube into the posteroinferior quadrant of the tympanic membrane under otomicroscopic view and fixed by a foam insert earphone (used for BAEP stimulation) as well as a cutaneous suture.

\section{FOLLOW UP}

Postoperatively, the patients were followed up on the intensive care unit. Brain CT, audiometry, BAEP recordings in those patients with preserved hearing, and a neurological examination were performed before discharge from hospital. After 6 weeks, 6 months, and at yearly intervals thereafter the patients were seen in the outpatient department for neurological status, audiometry, and MRI. A $t$ test was used for statistical analysis.

\section{Results}

SURGERY

A total of 508 patients were operated on by seven different neurosurgeons between April 1991 and December 1997. The annual rate of surgery increased from 45 in 9 months of 1991 and 61 in 1992 up to 89 in 1997 (fig 1). Facial nerve monitoring was introduced in

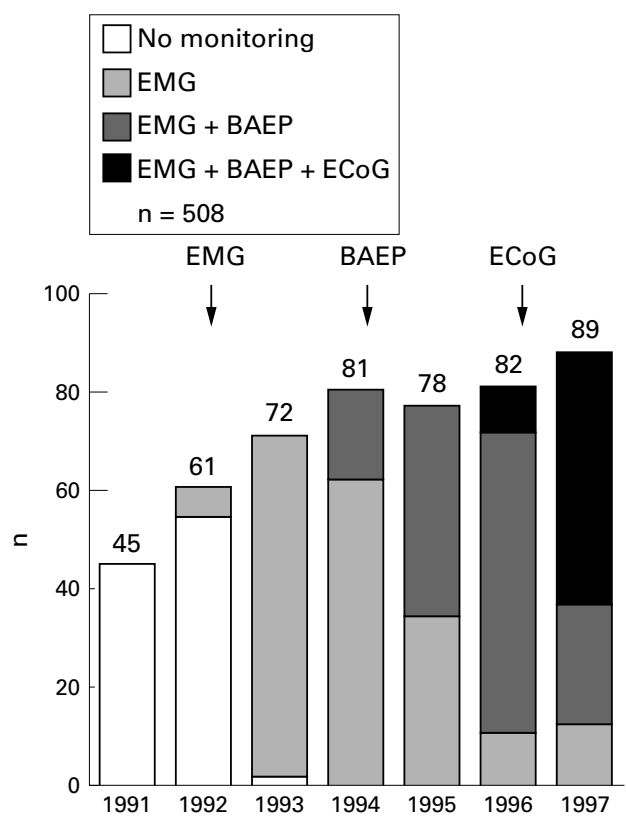

Figure 1 Yearly distribution of acoustic neuroma surgery at our institution with the proportion of the monitoring modalities used. 
Table 2 Distribution (\%) of the extrameatal size of acoustic neuromas

\begin{tabular}{lccc}
\hline & \multicolumn{2}{l}{$\begin{array}{l}\text { Patients with preserved } \\
\text { hearing }\end{array}$} \\
\cline { 3 - 4 } & All patients & Preop & Postop \\
\hline$\leqslant 15 \mathrm{~mm}$ & 51.6 & 54.5 & 66.2 \\
$16-30 \mathrm{~mm}$ & 42.4 & 41.6 & 33.8 \\
$>30 \mathrm{~mm}$ & 3.8 & 2.6 & 0 \\
No data & 2.2 & 1.3 & 0 \\
\hline
\end{tabular}

1992, intraoperative BAEP recording was added in 1994. Since 1996, intraoperative ECoG recording has been available at our institution.

The size of the extrameatal portion of the tumour (largest diameter) was up to $15 \mathrm{~mm}$ in $51.6 \%$ of patients, $42.4 \%$ had tumours with an extrameatal extension of $16-30 \mathrm{~mm}$ (table 2).

The most frequent complication was a subgaleal CSF collection in 47 patients $(9.2 \%)$, two of whom had to be corrected surgically. Leakage of CSF (mostly rhinoliquorrhea) occurred in 16 cases $(3.1 \%)$, all but six could be sufficiently treated by lumbar drainage for 3-5 days, the remainder had to be operated on. Twenty four patients $(4.7 \%)$ had cranial nerve deficits (besides functional impairment of the facial and the cochlear nerve), in six of them the function recovered within the first 6 months after surgery. The rate of meningitis was $2.9 \%$. Postoperative bleeding occurred in eight cases $(1.5 \%)$, six of these required reoperation. Wound healing was impaired in $0.5 \%$. In $5.6 \%$ of all procedures, an air embolism was detectable by means of either precardial Doppler sonography or end expiratory $\mathrm{pCO}_{2}$ decline. A clinically relevant air embolism event occurred only in $1.3 \%$. The overall mortality was $0.4 \%$ (one case each related to pulmonary embolism and myocardial infarction secondary to neurological deterioration).

As additional electrophysiological monitoring was considered to be time consuming, we analysed the time in the operating room dedicated to perioperative care including set up of the monitoring and the duration of the

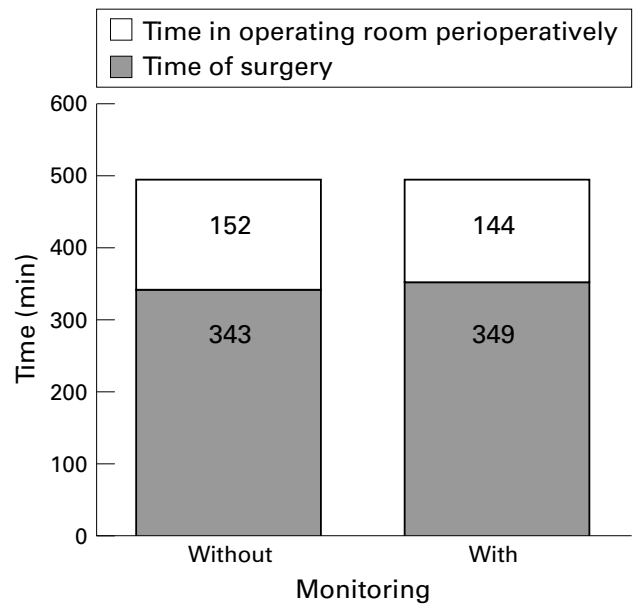

Figure 2 Mean time required ( $\mathrm{min}$ ) for acoustic neuroma surgery with and without intraoperative monitoring. Duration of the surgical procedure as well as perioperative management in the operating room (including set up of the monitoring) was similar in both groups.

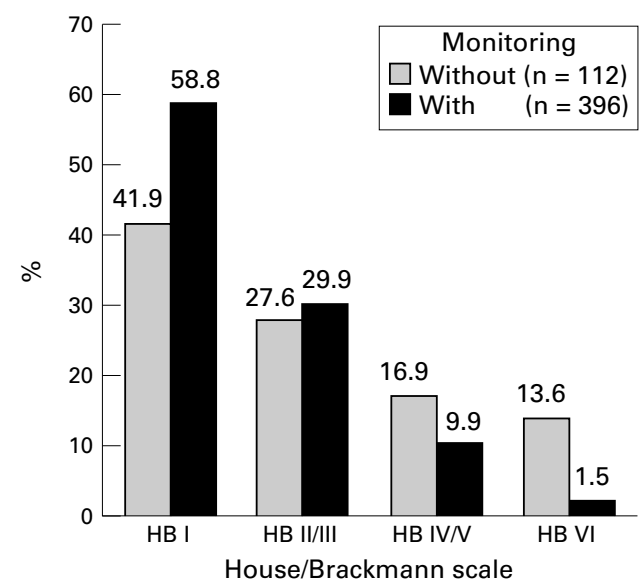

Figure 3 Functional outcome of facial nerve function 6 months after surgery in patients operated on with and without monitoring.

operation itself, comparing the cases between 1991 and 1992 without monitoring to those thereafter with at least one monitoring modality. Perioperative time and duration of surgery were similar in both groups (fig 2).

PRESERVATION OF FACIAL AND COCHLEAR NERVE FUNCTION

In 396 of 508 operations, location and function of the facial nerve were intraoperatively evaluated by means of EMG recording of the orbicularis oculi and orbicularis oris muscles. In $58.8 \%$ of these cases, no functional impairment of the facial nerve (House/Brackmann grade I) was detectable 6 months postoperatively. In $29.9 \%$, a slight (non-functional) deficit (House/Brackmann grade II) remained. Taken together, in $88.7 \%$ the preservation of facial nerve function was good or excellent. This compares favourably with $69.5 \%$ in those patients who had to be operated on before the introduction of intraoperative monitoring. The rate of complete nerve VII palsy (with no chance of a non-surgical rehabilitation) 6 months after surgery was reduced from $13.6 \%$ to $1.5 \%$ (fig 3 ).

Facial nerve function 6 months after surgery was correlated with tumour size. Although tumour size was related to clinical outcome for each individual surgeon, his surgical experience was neither reflected by this relation nor by the rate of preserved function.

Three hundred and ninety nine of the 508 patients presented with servicable hearing before surgery. As BAEP monitoring is only useful in patients with remaining cochlear function, only these 399 patients were suitable for BAEP monitoring. Since the introduction of intraoperative BAEP monitoring, 229 cases were operated on using the device. Thus we were able to compare our results on functional hearing in patients with $(n=229)$ and without $(n=170)$ BAEP monitoring. Good functional hearing with a hearing loss in pure tone audiometry of less than $50 \mathrm{~dB}$ (modified Gardner/Robertson grades 1 and 2) could be achieved in $26.8 \%$ of the monitored group compared with only $8.8 \%$ without BAEP monitoring. Serviceable hearing (modified 


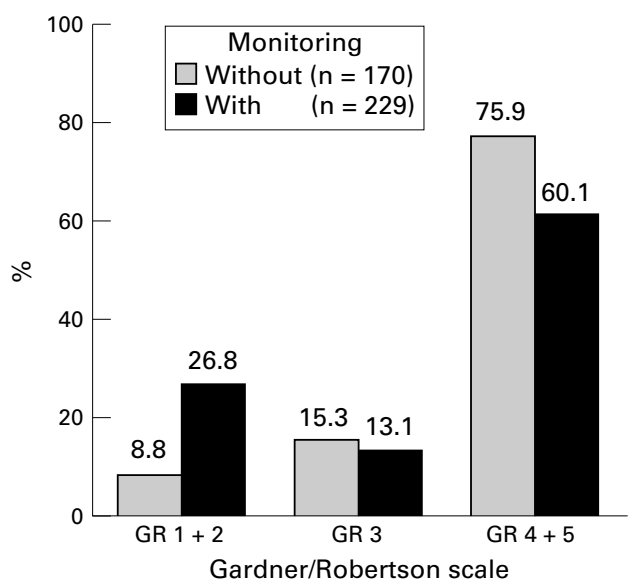

Figure 4 Hearing preservation 6 months after surgery in patients operated on with or without monitoring.

Gardner/Robertson 1-3 as mostly defined in other studies) was obtained in $38.9 \%$. Little or no cochlear function remained in $60.1 \%$ of the monitored and in $75.9 \%$ of the non-monitored cases (fig 4). Hearing preservation was correlated with tumour size in that in larger tumours (diameter $>30 \mathrm{~mm}$ ) we could not achieve results better than Gardner/Robertson 4 postoperatively (table 1). Interestingly, the size distribution of tumours in patients with preserved hearing preoperatively did not differ from the overall group (table 2 ).

In 49 patients, intraoperative BAEP and ECoG monitoring were performed in parallel. We compared the electrophysiological data with the pure tone audiometry 1 week after surgery; BAEP was considered preserved when at least one of the peaks III - V was maintained additionally to the waves I and II. Twenty two of 26 patients with preserved BAEP and ECoG had good functional hearing (modified Gardner/Robertson 1-3) postoperatively. All patients with a loss of the ECoG signal $(n=18)$ at the end of surgery were deaf postoperatively. One patient with maintained ECoG and loss of BAEP intraoperatively displayed hearing sensations according to Gardner/Robertson grade $3(50-90 \mathrm{~dB})$ in pure tone audiometry after surgery, another had to be classified as grade 4 $(>90 \mathrm{~dB})$. Three patients with this constellation were deaf. In conclusion, these two patients had preserved hearing sensations despite the loss of the BAEP waves III-V, a phenomenon described as "disconnected ear". ${ }^{4}$

\section{Discussion}

FACIAL NERVE FUNCTION

The remarkable improvements in diagnostic tools, such as MRI and neurophysiology, permit the more precise diagnosis of an acoustic neuroma and, moreover, at an earlier stage of the disease. Consequently, the functional performance of the facial nerve and hearing ability in these patients at the time of diagnosis is now better compared with former times. ${ }^{5}$ Hence, functional preservation has become the major goal in acoustic neuroma surgery today. For the facial nerve, anatomical preservation has become the rule and disruption the exception, disruption mostly occurring in the treatment of recurrences or very large tumours, especially in patients with neurofibromatosis-2 (the rare facial neuroma with its high inherent surgical vulnerability of the facial nerve is not the subject of this paper). Several series of patients operated on for acoustic neuromas have been published in the past years. The largest summarised data from 1400 patients over 26 years performed in numerous centres, ${ }^{6}$ 1000 patients/ 15 years being operated on by a single surgeon, ${ }^{78} 611$ patients $/ 21$ years, ${ }^{9} 514$ patients/ 12 years, ${ }^{10} 476$ patients/ 16 years, ${ }^{11} 335$ patients/11 years, ${ }^{12} 255$ patients $/ 8$ years, ${ }^{13}$ and 179 patients $/ 2$ years, ${ }^{14}$ compared with our series of 508 consecutive patients over a 7 year period. In the literature the rate of a good postoperative function of the facial nerve (House/ Brackmann grades I and II) is reported to range from $59 \%$ to $93 \% .^{6}{ }^{7-14}$ There is a clear cut correlation with tumour size and, moreover, a significant increase in the functional preservation rate over the past decades, mainly due to intraoperative monitoring of the facial nerve, which is now recommended by the National Institute of Health (Consensus Development Conference on Acoustic Neuroma, 1991)..$^{15}$ The bipolar stimulation of the cranial nerves during surgery enables the surgeon to localise the nerve at an early stage of the surgical procedure, to distinguish the structure from tumour tissue, and to determine its functional integrity. Comparing our own results with and without monitoring of nerve VII, a dramatic functional improvement was achieved with monitoring: facial nerve function was normal (House/Brackmann I) in $58.8 \%$ with, but only in $41.9 \%$ without monitoring, considering the overall group including all tumour sizes. Indeed tumour size cannot account for the improvement of the functional outcome as there was no significant change in mean tumour size comparing the first 2 years (without monitoring of the facial nerve) and the years thereafter. However, there was a slight trend towards smaller tumours with increasing availability of MRI. Individual surgical experience does not seem to be an explanation as all operations in 1991 and 1992 were performed by senior staff members with long experience in acoustic neuroma surgery-the younger surgeons joined the group after the introduction of facial nerve monitoring (which was considered to offer additional safety for the surgeons in training). Summing up the rate of preserved facial nerve function with a good prognosis for further rehabilitation 6 months postoperatively, House/Brackmann grades I-III could be achieved in $88.7 \%$ with monitoring versus $69.5 \%$ without. The proportion of cases with complete palsy of the facial nerve without any chance for further improvement apart from surgical reconstructive measures has dropped from 13.6 to $1.5 \%$ since the introduction of facial nerve stimulation. These data compare favourably with other reported large series. 
HEARING PRESERVATION

In the past 10 years the preservation of functional hearing in acoustic neuroma surgery has increasingly become a focus of treatment. In patients with good hearing sensation (modified Gardner/Robertson grades 1-3) preoperatively, rates of hearing preservation are reported between $13.5 \%$ and 52\%. ${ }^{6}{ }^{10-14}{ }^{16-19}$ Two of these series, however, with $50 \%$ of preserved hearing, include a high proportion of intracanalicular tumours, in which the preservation of cochlear function is far better. ${ }^{16}{ }^{17}$ As all 508 patients presented in this series presented with intrameatal and extrameatal tumour extension (true intracanalicular neuromas are operated on in the ENT department at our university and are not included in this report), our rate of $39.9 \%$ of preserved hearing (modified Gardner/Robertson grades 1-3) compares favourably with the data from recent literature.

Monitoring of BAEPs taught us much concerning intraoperative handling of the cochlear nerve: during on line monitoring, wave III and V of the BAEPs were extremely sensitive to surgical manipulation. We found distension of the nerve to be more dangerous for the function of the cochlear nerve than compression. Reducing the drill speed to below $10000 \mathrm{rpm}$ when opening the fundus of the inner auditory canal was helpful in preserving good BAEPs as was avoiding bipolar coagulation in the vicinity of the cochlear nerve. Instead, continuous irrigation with Ringer's solution seemed safer. This is, however, only an alternative in the semisitting position which we favour routinely, as the irrigant spills from the craniotomy, providing an optimal exposure all the time. Many of these experiences have been recently confirmed in an extensive personal series of 1000 patients with acoustic neuroma over 14 years. ${ }^{520}$ Once the wave III or V of the BAEPs declined or its latency increased, a short pause during dissection of the neuroma or drilling the petrous bone increased the likelihood of signal recovery. Thus continuous BAEP recording has become the "surgeon's guide" during these procedures. However, sometimes the BAEP waves III-V were suddenly lost during preparation. We considered this rapid "apoplectiform" loss of cochlear function to be damage of vascular structures of the cochlear nerve or the cochlea itself. In these instances, recovery of cochlear function was extremely rare by contrast with a slow ceasing of the interpeak latencies or the amplitudes of BAEP waves III or V.

Electrocochleography has been used since 1996 as a supplementary tool for intraoperative monitoring of hearing. Compared with BAEP, ECoG provides some distinct advantages, as it can even be recorded during critical manoeuvres such as electrocautery and drilling of the petrous bone without requiring averaging. It has been argued that ECoG-since it is generated in the cochlea and the intracanalicular portion of the VIIIth nerve-does not reflect damage to the auditory nerve during cerebellopontine angle surgery and might thus not be suitable for intraoperative monitoring of acoustic neuromas. ${ }^{21}$ However, one rationale for intraoperative ECoG recordings can be derived from experimental observations in animals which show manipulations in the cerebellopontine angle to cause an avulsion rupture of the internal auditory artery followed by an immediate extinction of all BAEP signals. ${ }^{22}$ Moreover, our data from patients with combined $\mathrm{BAEP} / \mathrm{ECoG}$ recordings indicates the presence or absence of the ECoG action potential to be at least as well correlated to postoperative hearing as any indices of BAEP. Altogether, ECoG presents a reliable tool for intraoperative monitoring of hearing during acoustic neuroma surgery. Its applicability, however, is restricted to manipulations of the intracanalicular and distal extracanalicular portion of the VIIIth nerve. Thus, ECoG should be regarded as a supplementary method, but not a substitute, for BAEP monitoring. ${ }^{3}$

\section{SURGICAL CONCEPT}

The results of facial nerve preservation and the functional outcome in our series support the concept of an interdisciplinary neurosurgical/ ENT service for acoustic neuroma surgery. The neurosurgeon has the chance to share the expertise of the ENT surgeon in drilling the petrous bone by noting the anatomical landmarks (for instance, the ductus endolymphaticus, semicircular canal) which are essential in preserving the cochlear structures in cases with persistent hearing ability. In cases of a loss of the BAEP signal intraoperatively we jointly try to preserve cochlear structures as well as the cochlear nerve to maintain the patient's option of receiving a cochlear implant later should this become necessary (for example, in case he becomes deaf on the other ear as well).

In terms of overall complications, our data do not differ from other large series, with CSF leaks being the most frequent $(12.3 \%$ in our series compared with $9.2 \%-15 \%$ in others). ${ }^{11} 131420$ Although a "learning curve" had to be anticipated for each neurosurgeon at the beginning, the comparative analysis of complications did not show that most problems were encountered by the "beginners". Instead, we found a homogeneous distribution for each surgeon with no one exceeding the complication rate described in other large series, even those treated by one surgeon. ${ }^{20}$

\section{Conclusion}

Acoustic neuroma surgery via a retrosigmoidal approach is a safe and effective treatment for tumours with extrameatal extension. Intraoperative monitoring of cranial motor nerves (especially the facial nerve) and continuous BAEP recording substantially improve functional outcome. We found the interdisciplinary neurosurgery/ENT concept convincing as each step of the operation is performed with subspecialty expertise.

The technical assistance of Ms E Hoffmann in all cases with intraoperative monitoring is highly appreciated as well as the stylistic correction of the manuscript by Dr GH Vince. Parts of this study have been presented at the 48th Annual Meeting of the German Neurosurgical Society in Berlin, 27-30 April 1997.

1 Gardner G, Robertson JH. Hearing preservation in unilateral acoustic neuroma surgery. Ann Otol Rhinol Laryngol 1988;97:55-66. 
2 House JW, Brackmann DE. Facial nerve grading system. Otolaryngol Head Neck Surg 1985;93:146-7.

3 Schlake HP, Goldbrunner RH, Milewski C, et al. Technical developments in intra-operative monitoring for the preservation of cranial motor nerves and hearing in skull base surgery. Neurol Res 1998;21:11-24.

4 Cacace AT, Parnes SM, Lovely TJ, et al. The disconnected ear: phenomenological effects of a large acoustic tumor. Ear Hear 1994;15:287-98.

5 Matthies C, Samii M. Management of vestibular schwannomas (acoustic neuromas): the value of neurophysiology for evaluation and prediction of auditory function in 420 cases. Neurosurgery 1997;40:919-29.

6 Sterkers JM, Morrison GA, Sterkers O, et al. Preservation of facial, cochlear, and other nerve functions in acoustic neuroma treatment Otolaryngol Head Neck Surg 1994;110: 146-55.

7 Samii M, Matthies C. Management of 1000 vestibular schwannomas (acoustic neuromas): the facial nerveschwannomas (acoustic neuromas): the facial nerve1997;40:684-94.

8 Samii M, Matthies C, Tatagiba M. Management of vestibular schwannomas (acoustic neuromas): auditory and facial nerve function after resection of 120 vestibular schwannomas in patients with neurofibromatosis 2. Neurosurgery 1997;40:696-70

9 Sampath P, Holliday MJ, Brem H, et al. Facial nerve injury in acoustic neuroma (vestibular schwannoma) surgery: etiology and prevention. F Neurosurg 1997;87:60-6.

10 Rowed DW, Nedzelski JM. Hearing preservation in the removal of intracanalicular acoustic neuromas via the retrosigmoid approach. F Neurosurg 1997;86:456-61.

11 Cohen NL, Lewis WS, Ransohoff J. Hearing preservation in cerebellopontine angle tumor surgery: the NYU experience 1974-91. Am f Otol 1993;14:423-33.
12 Harner SG, Beatty CW, Ebersold MJ. Retrosigmoid removal of acoustic neuroma: experience 1978-1988 [comments]. Otolaryngol Head Neck Surg 1990;103:40-5.

13 Ebersold MJ, Harner SG, Beatty CW, et al. Current results of the retrosigmoid approach to acoustic neurinoma [comments]. F Neurosurg 1992;76:901-9.

14 Gormley WB, Sekhar LN, Wright DC, et al. Acoustic neuromas: results of current surgical management. Neurosurgery 1997;41:50-8.

15 Kartush JM. Intra-operative monitoring in acoustic neuroma surgery [in process citation]. Neurol Res 1998;20: 593-6.

16 Irving RM, Jackler RK, Pitts LH. Hearing preservation in patients undergoing vestibular schwannoma surgery: comparison of middle fossa and retrosigmoid approaches. $\mathcal{F}$ Neurosurg 1998;88:840-5.

17 Kanzaki J, Ogawa K, Inoue Y, et al. Quality of hearing preservation in acoustic neuroma surgery [in process citation]. Am f Otol 1998;19:644-8.

18 Post KD, Eisenberg MB, Catalano PJ. Hearing preservation in vestibular schwannoma surgery: what factors influence outcome? [comments]. F Neurosurg 1995;83:191-6.

19 Samii M, Matthies C. Management of 1000 vestibular schwannomas (acoustic neuromas): hearing function in 1000 tumor resections. Neurosurgery 1997;40:248-60.

20 Samii M, Matthies C. Management of 1000 vestibular schwannomas (acoustic neuromas): surgical management and results with an emphasis on complications and how to avoid them. Neurosurgery 1997;40:11-21.

21 Moller AR. Neurophysiological monitoring in cranial nerve surgery. Neurosurg Quart 1995;5:55-72.

22 Sekiya T, Moller AR. Avulsion rupture of the internal auditory artery during operations in the cerebellopontine angle: a study in monkeys. Neurosurgery 1987;21:631-7. 